\title{
Making your presentation
}

\section{communicate}

\author{
By Mignon Adams
}

Director of Library Services

Philadelphia College of Pharmacy and Science

\section{It's not enough to "stand and deliver."}

ike me, you have probably attended many presentations made by librarians at professional meetings. You may have suffered through the person who stands at a podium reading and droning through a prepared script, filled with figures impossible to understand. You may also have been asked to look at an overhead transparency you couldn't read because it was filled with minuscule type. Or you may have been shown murky slides whose subjects you couldn't determine.

Perhaps, like me, you have accepted the status quo of poor professional library presentations, placing the blame on badly designed rooms, the seriousness of the subject matter, and long tradition. However, I recently began attending the national meetings of an organization whose members (and presenters) are primarily scientists. I found that what librarians accept as the norm for presentation is simply not found at scientific meetings.

At almost every session I attended, whether it was in a ballroom or a small conference room, the presenters used some type of visuals. There were no overheads with teeny-tiny type, and no dimly lit slides; instead, the visuals were legible, crisp, and professional. Complex scientific information and tables were presented graphically in a way that even I, a nonscientist, could comprehend them. The only exceptions to the rule that speakers made very good visual presentations were one librarian and one speaker from the humanities.

Librarians should emulate our scientific col- leagues. We should differentiate between "delivering a paper" and "making a presentation." Professionals in other disciplines who truly want to communicate plan a total presentation instead of merely writing a paper. To do so requires some advance thinking and preparation.

One problem that requires thought is that the presenters at a professional meeting rarely know ahead of time the specifics of the room they will be using. Hotel ball rooms were not designed for largegroup presentations. The chance of a poorly configured room must be kept in mind as visuals are planned.

Three kinds of visuals are commonly used to accompany presentations: handouts, overheads, and slides. Handouts are easy to produce and require no special equipment beyond a typewriter and a photocopier. If you will be discussing numbers or any other information which can be more easily grasped through a graphic display, then handouts provide a convenient way to make sure your audience can follow you. Many people appreciate an outline of your talk or a list of major points. You may lose a "surprise" factor-but suspense and surprise usually play a small part in a professional presentation. Finally, handouts work well no matter what size or shape your assigned room might turn out to be.

Overheads are also easy to produce. Almost any standard photocopier will make good transparencies. Because they are so easily produced, overhead 
transparencies are perhaps the most misused visual aid. Regular-sized typing on a transparency cannot be read by anyone in an audience more than about seven feet away. We have all been forced to view an entire typed page which has simply been photocopied onto a transparency - and we have all resented it. Pages photocopied from a book can be even worse. Letters on a transparency should be at least $1 / 4$ high ( 18 to 24 points) to be read by an audience of more than ten people. Normal typing is about $1 / 8$ (10 to 12 points).

It is easy to enlarge your copy before you make a transparency. Enlarging photocopiers are now fairly common. Many of us have access to wordprocessors which will create larger print, and software packages especially designed for creating transparency masters are available.

Even if you have no equipment for enlargement, you can vastly improve over typewriter size by hand printing your information onto an acetate sheet with a felt-tip marker (one designed for overheads, available from office supply stores). Your transparencies may lack professional polish-but at least your audience will be able to read them.

Overhead transparencies are an excellent medium to use in a classroom-sized or seminar room, since lights can be kept on and a plain sheet of acetate can be used as a substitute for a chalkboard. However, overheads can be a poor choice for a large room such as a ballroom or auditorium. In order for the image to be large enough, the overhead often must be placed at some distance from the speaker, who then must rely on someone else to manipulate the materials. If the room is exceptionally wide, those seated on the sides will be unable to see the screen. In very large rooms, overheads should be accompanied by handouts, or the better choice may be slides. Nearly all convention hotels and centers can provide a high quality slide projector, with a remote control at the podium, and in a set-up which can reasonably be viewed by the entire audience.

To avoid speaking in a dark room, presenters may choose two types of fairly inexpensive slides which can be seen with the lights left on. One is produced by orthographic film (also called Kodalith, a brand name, or high contrast). Orthographic slides have a black background with light coming through the photographed lines. These slides are bright enough to be seen with all the lights on. Vericolor slides, made with a recently developed film, produce bright white lines on a vivid blue background. Lights may be left on with these slides, but may need to be dimmed slightly.

Both orthographic and Vericolor slides photograph only lines. They will reproduce type, charts, tables, and line drawings (such as cartoons) well, but not photographs. With a little practice and a copy stand, an amateur photographer can produce good images on these films. However, the processing is tricky and should probably be left to a professional photographer or commercial processor. If your campus has a photography unit, the people there will know about these slides.

Some speakers can keep an audience spellbound without using any visual help whatsoever, but most of us are not natural orators. Planning and using visuals may take more time and more money-but these are small increases compared to the time and money you and your institution are already investing in your presentation. I, and everyone else in your audience, will be very appreciative.

\section{Attention, authors!}

\section{College \& Research Libraries News wel} comes manuscripts submitted on diskette or via ALANET. The C $r R L$ News ALANET number is ALA0306. If you can upload it, we can download it, because ALANET translates text into pure ASCII files. If you don't have ALANET or you prefer to send a diskette, make sure it is formatted for MS-DOS; either 5.25-inch or 3.5inch diskettes can be accepted.

We use Xywrite III + forword processing, but we transiate files from other major programs. When submitting a diskette with a file created by another word processing program, it is best to also include an ASCII text file. Also, please specify which word processing program you have used; we can convert them if we know what we are converting from.

Always send a paper copy of your manuscript along with the diskette, just in case we cannot read your file.

If you can neither send a diskette nor transmit via ALANET, submit three paper copies in a standard typeface such as Courier or Pica. Please avoid sending proportional or oversize typefaces. Do not fax articles unless you are requested to do so.

Queries are not required. If you need clarification on the appropriateness of your topic, please call rather than write. Notification of acceptance or rejection will be made within four to six weeks

Submit all materials to Editor, C $b R L$ News, ACRLALA, 50 E. Huron St., Chicago, IL 60611; (312) 280-3511.

\section{Attention serials librarians}

The C\&RL News annual index for 1990 appears in this issue. 